\title{
3D EMHD reconnection in a laboratory plasma
}

\author{
R. L. Stenzel, J. M. Urrutia, M. C. Griskey, and K. D. Strohmaier \\ Department of Physics and Astronomy, University of California, Los Angeles, CA 90095-1547, U.S.A.
}

(Received June 1, 2000; Accepted July 11, 2000)

\begin{abstract}
In a large laboratory plasma, reconnection of three-dimensional (3D) magnetic fields is studied in the parameter regime of electron magnetohydrodynamics (EMHD). The field topologies are spheromak-like with twodimensional null lines and three-dimensional spiral null points. The relaxation of an initial vortex field by spontaneous reconnection is studied in the absence of boundary effects. Reconnection rates and energy conversion from fields to particles are measured. The frozen-in condition appears to be destroyed by viscous effects rather than inertia or collision. Finally, the non-driven merging of two EMHD spheromaks into a long-lived FRC is observed. These basic physics experiments demonstrate that reconnection is an important process in the parameter regime of unmagnetized ions, which is always encountered near absolute magnetic null points.
\end{abstract}

\section{Introduction}

Electron magnetohydrodynamics (EMHD) describes the plasma parameter regime of magnetized electrons and unmagnetized ions (Kingsep et al., 1990). Such conditions occur on small scale lengths $\left(r_{c e}<r<r_{c i}\right)$ and short time scales $\left(t_{c e}<t<t_{c i}\right)$. In this regime the magnetic field is frozen into the electron fluid, provided resistivity, viscosity, and inertia are negligible (scale $\left.>c / \omega_{p e}\right)$. Just like in MHD, magnetic field line reconnection can occur near magnetic null points where EMHD breaks down. Reconnection can change the field topology, lead to transport of electrons across the magnetic field, and energization of particles on expense of magnetic field energy. In MHD the jetting of a charge-neutral fluid constitutes an irreversible energy conversion process. However, in EMHD, jetting of electrons produces currents and magnetic fields such that no significant loss of magnetic energy occurs. It requires randomization processes to convert magnetic energy into electron heat. Although in EMHD the ions are unmagnetized, they are not necessarily immobile. If reconnection takes place slowly $\left(\omega_{p i}^{-1}<t \ll \omega_{c e}^{-1}\right)$, space charge electric fields can accelerate the ions, thus magnetic field energy can be transferred to both electrons and ions (Stenzel et al., 1982).

The physics of magnetic field line reconnection has originally been developed for two-dimensional (2D) fields, $\mathbf{B}(x, y)$, with a null in one, two, or three field components (Vasyliunas, 1975). Of course, most geometries in nature are three-dimensional (3D), thus the present focus has shifted to $3 \mathrm{D}$ reconnection problems. Common to all reconnection geometries is the formation of localized current layers whose strength and size determine the flux transfer across the separatrix, i.e., the reconnection rate. Strong current sheets are thought to provide possible mechanisms for breaking the frozen-in condition by enhancement of turbulent resis-

Copy right (c) The Society of Geomagnetism and Earth, Planetary and Space Sciences (SGEPSS); The Seismological Society of Japan; The Volcanological Society of Japan; The Geodetic Society of Japan; The Japanese Society for Planetary Sciences. tivity, viscosity, and inertia. Of course, the frozen-in condition is always violated in absolute magnetic null points. Absolute null points in 3D can be characterized by a spine and fan geometry (Parnell et al., 1996), which are extensions of 2D X- and O-type null points. A great variety of fan-spine nulls has been theoretically analyzed and categorized. Here we will report direct experimental measurements of spiral null points in a laboratory plasma. They are observed in connection with $3 \mathrm{D}$ vortices in the electron fluid. Such Hill's type vortices generate spheromaklike magnetic fields, which are superimposed on a uniform background magnetic field $\mathbf{B}_{0}$. The vortices are induced with pulsed currents through a loop antenna. When the vortex fields are smaller than the background field, the total field exhibits a twist and bulge/pinch but no null points. This is the linear regime of EMHD vortices which has been studied extensively (Stenzel and Urrutia, 1990; Rousculp et al., 1995; Urrutia et al., 2000). A brief summary of their interesting properties will be given prior to describing new observations of large-amplitude vortices with 3D null points. The latter are formed when the dipole field of the loop antenna opposes and exceeds the background magnetic field. After establishing a quasi steady-state potential field with two cusp-type null points in a high-beta plasma $\left[\beta=n k T_{e} /\left(B^{2} / 2 \mu_{0}\right) \approx 1\right]$, the source current in the antenna is abruptly switched off and the free relaxation of the initial magnetic field topology is observed in an essentially unbounded uniform EMHD plasma. It involves spontaneous 3D reconnection whereby the "closed" dipolar magnetic field lines are reconnected into "open" field lines. In contrast to most previous reconnection experiments, there is no external energy source driving the reconnection, there are no currents in rigid coils or conducting boundaries, all fields and currents evolve self-consistently as they would in an unbounded space plasma of similar parameters, for example, the solar photosphere. Of course, whether these processes actually take place in the photosphere cannot be con- 


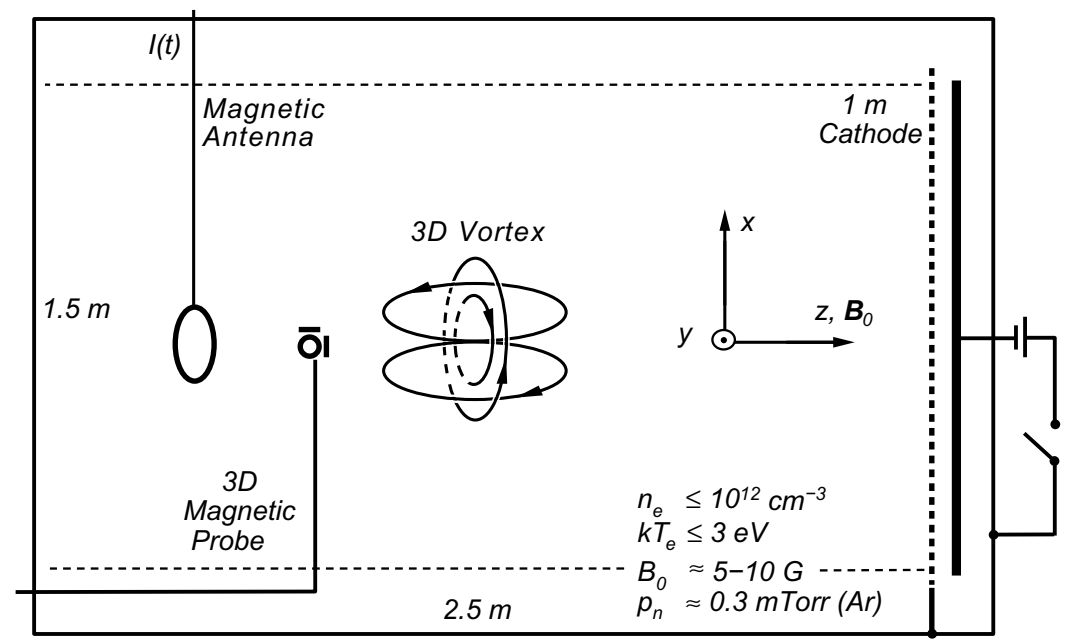

Fig. 1. Experimental setup and basic plasma parameters.

firmed since present solar observations lack the required resolution by six orders of magnitude. From the laboratory observations we obtain the relaxation, i.e., reconnection, which demonstrates that magnetic energy is converted into electron heat and light. Furthermore, we study the spontaneous interaction of two nonlinear vortices of opposite helicity, which are observed to merge into a field-reversed configuration, FRC (Ji et al., 1998). These laboratory experiments address the basic physics of $3 \mathrm{D}$ reconnection in the new parameter regime of EMHD, which should be relevant to all absolute magnetic nulls at distances $r_{c e}<r<r_{c i}$ from the null point.

The paper is organized as follows: After describing in Section 2 the plasma device and measurement techniques, the experimental results are presented in Section 3, divided into various subsections. The conclusion, Section 4, points out the relevance of the present findings to related observations and applications.

\section{Experimental Arrangement}

The experiments are performed in a large laboratory plasma device schematically shown in Fig. 1. A $1 \mathrm{~m}$ diam $\times$ $2.5 \mathrm{~m}$ long plasma column of density $n_{e} \leq 10^{12} \mathrm{~cm}^{-3}$, electron temperature $k T_{e} \leq 3 \mathrm{eV}$, Argon gas pressure $p_{n} \simeq$ 0.3 mTorr, is produced in a uniform axial magnetic field $B_{0} \simeq 5-10 \mathrm{G}$ with a pulsed dc discharge $\left(V_{\text {dis }} \simeq 50 \mathrm{~V}\right.$, $I_{\text {dis }} \simeq 600 \mathrm{~A}, t_{\text {pulse }} \simeq 5 \mathrm{~ms}, t_{\text {rep }} \simeq 1 \mathrm{~s}$ ) using a large oxidecoated cathode. Pulsed currents are applied to insulated magnetic loop antennas $(1 \ldots 15 \mathrm{~cm}$ diam, $1 \ldots 4$ turns $)$ in the steady-state discharge plasma or the early current-free afterglow. The time-varying magnetic fields produced by the applied antenna and induced plasma currents are measured with a triple magnetic probe, recording $\left(B_{x}, B_{y}, B_{z}\right)$ versus time at a given position. By repeating the highly reproducible discharges and moving the probe to many positions in a three-dimensional volume, the vector field $\mathbf{B}(\mathbf{r}, t)$ is obtained with high resolution $(\Delta r \simeq 0.75 \mathrm{~cm}, \Delta t \simeq$ $10 \mathrm{~ns})$. At any instant of time, the spatial field distribution can be constructed from the digitally stored temporal traces. The current density is calculated from Ampère's law, $\mathbf{J}=\nabla \times \mathbf{B} / \mu_{0}$ without making any assumptions of field
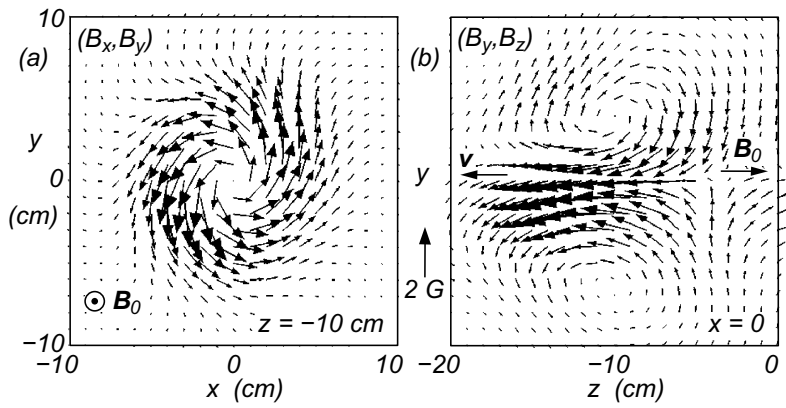

Fig. 2. (a) Toroidal and (b) poloidal magnetic field components of a small amplitude vortex propagating in the whistler mode along a stronger uniform magnetic field $B_{0}=5 \mathrm{G}$.

symmetries. Plasma parameters are obtained from a small Langmuir probe $\left(\pi r^{2} \simeq 2.6 \mathrm{~mm}^{2}\right)$, which is also movable in three dimensions.

\section{Experimental Results}

\subsection{Review of linear EMHD vortices}

When a current pulse $\left[\omega_{c e}^{-1}<\left(t_{\text {rise }}, t_{\text {fall }}\right)<\omega_{c i}^{-1}\right]$ is applied to a loop antenna with axis along $\mathbf{B}_{0}$, time-dependent plasma currents are induced that control the field penetration into the plasma. These currents consist of a toroidal electron Hall current and linked poloidal electron currents. The latter form two opposing dipolar current loops on either side of the loop antenna, which is due to the adiabatic $\mathbf{E} \times \mathbf{B}$ drift of the incompressible electrons in the time-varying dipole field. Ions form a stationary background population. Only very small charge imbalances are needed $\left(\left|n_{i}-n_{e}\right| / n_{e} \approx 10^{-6}\right)$ to produce the radial electric fields sufficient to drive the toroidal Hall current. The linked currents or electron fluid drifts form two 3D vortices, which propagate away from the loop antenna. For one of these vortices, the measured linked magnetic field components are displayed in Fig. 2. The propagation is predominantly along $\mathbf{B}_{0}$ in the whistler mode (Urrutia et al., 1994). Thus, the penetration of the potential field of the antenna is not controlled by diffusion, but by convection at the whistler speed. 
(a)
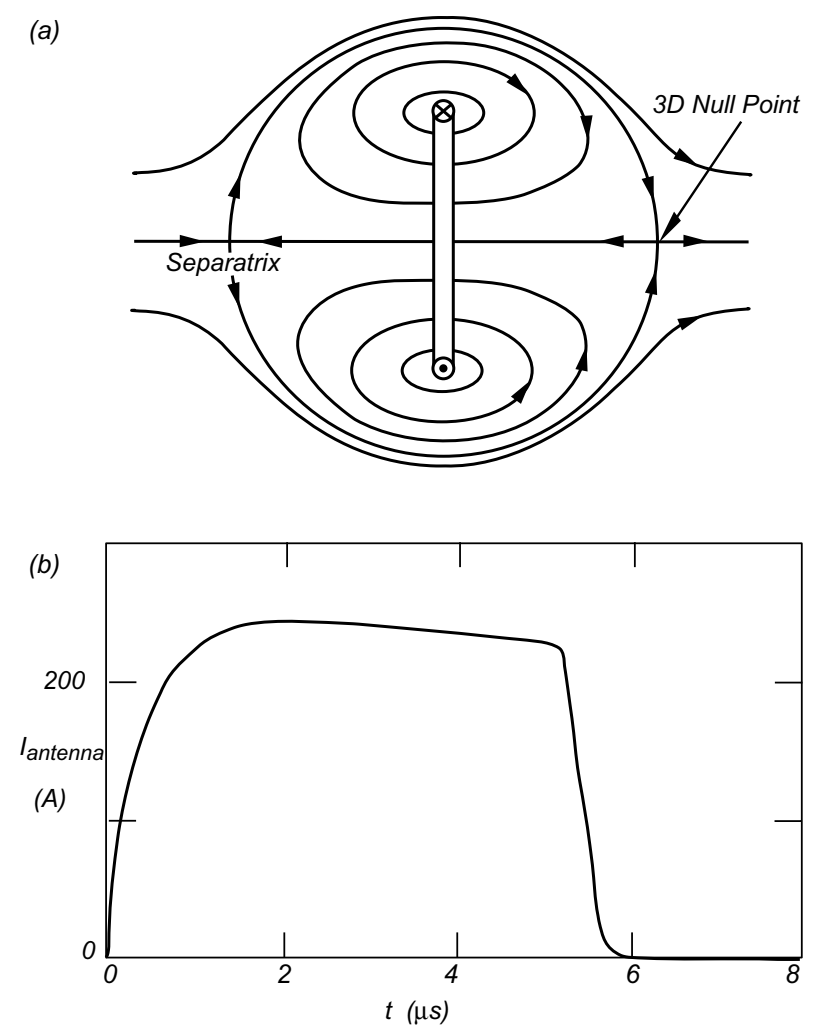

Fig. 3. (a) Schematic field topology of a strong dipole field, created by a current in a wire loop, in an opposing uniform background magnetic field. (b) Experimentally applied current to the loop antenna.

A transient EMHD vortex has positive (negative) helicity for propagation along (opposite to) $\mathbf{B}_{0}$. Upon normal reflection from a highly conducting boundary the magnetic helicity (self and mutual) changes sign while the energy is conserved. This result appears to violate helicity conservation but is explained by a break-down of the frozen-in condition at the electron-depleted plasma-boundary interface (Stenzel et al., 1999). Thus, field-line tying does not hold in EMHD. Within the plasma volume the magnetic self-helicity $\int \mathbf{A}_{\text {vortex }} \cdot \mathbf{B}_{\text {vortex }} d V$ and energy $\int B_{\text {vortex }}^{2} d V$ are found to decay at the same rate. However, the mutual helicity, $\int \mathbf{A}_{0} \cdot \mathbf{B}_{\text {vortex }} d V$, scales linearly with vortex field, hence is better conserved than the magnetic energy, which decays as the square of the vortex field. A useful example of helicity conservation is the injection of helicity from a suitable antenna such as a wire loop linked through a torus (Stenzel and Urrutia, 1999). Such helicity injection produces a unidirectional excitation of EMHD vortices. Transmission between two helicity antennas is unidirectional and non-reciprocal.

In EMHD, Ohm's law is dominated by the Hall effect, $\mathbf{E}+\mathbf{v} \times \mathbf{B}_{\text {total }} \simeq 0$, which implies force-free electromagnetic fields, $-n e \mathbf{E}+\mathbf{J} \times \mathbf{B}_{\text {total }} \simeq 0$. Since such fields exert no force on the electron fluid, they behave remarkably linear. No nonlinear interaction is observed when two EMHD vortices propagate along $\pm \mathbf{B}_{0}$ against each other (Urrutia et al., 2000). During the collision, the opposing (e.g., toroidal) fields annihilate. But, in contrast to MHD field line annihilation, the associated magnetic energy is not lost but converted via electron acceleration and currents into the linked

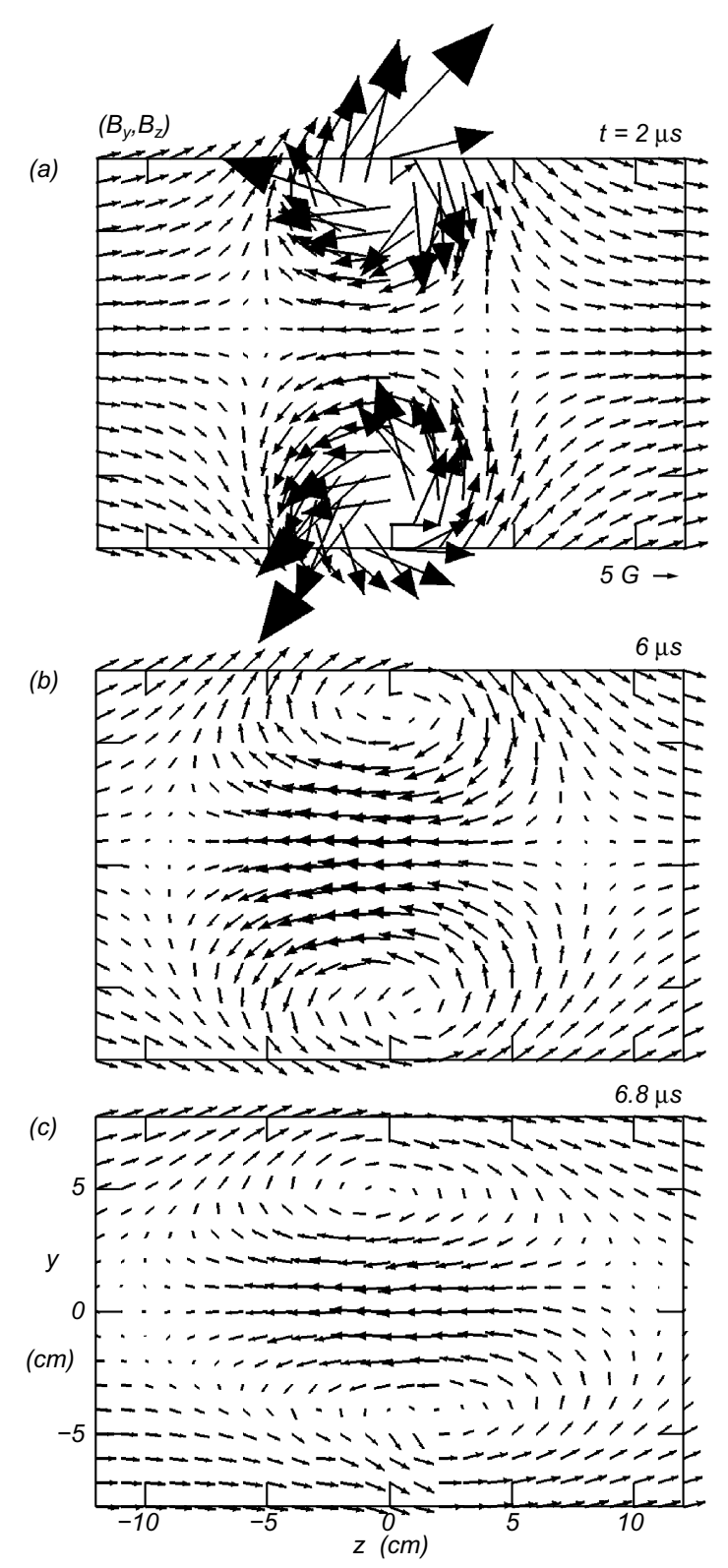

Fig. 4. Measured magnetic field vectors $\mathbf{B}_{t o t}$ (a) during the antenna current pulse, (b) and (c) after the end of the antenna current pulse. In the latter case the vortex is maintained by plasma currents driven by the free energy of the magnetic vortex. The relaxation involves spontaneous reconnection of closed to open field lines which is completed within $5 \mu \mathrm{s}$ after switch-off. No stabilizing currents are induced in rigid boundaries, yet the vortex does not tilt appreciably.

(e.g., poloidal) magnetic field energy. The energy transfer is reversed after the collision. Thus, energy and zero net helicity are conserved and the two-component EMHD field line "annihilation" did not lead to a breakdown of the frozen-in condition, i.e., reconnection.

If the electron inertia is retained in Ohm's law, the generalized vorticity, $\Omega=\mathbf{B}+\left(c / \omega_{p e}\right)^{2} \nabla^{2} \mathbf{B}$ is frozen into the electron fluid, $\partial \Omega / \partial t=\nabla \times(\mathbf{v} \times \Omega)$, allowing for slippage of the magnetic field lines through the fluid. This occurs for vortices of scale lengths $\leq c / \omega_{p e}$. Attempts to generate such small vortices with antennas have not been successful so far. Likewise, EMHD vortices have not been produced in the inertial regime $\left(\right.$ scale $<r_{c e}=v_{t h} / \omega_{c e}$ ). Earlier experi- 
(a)

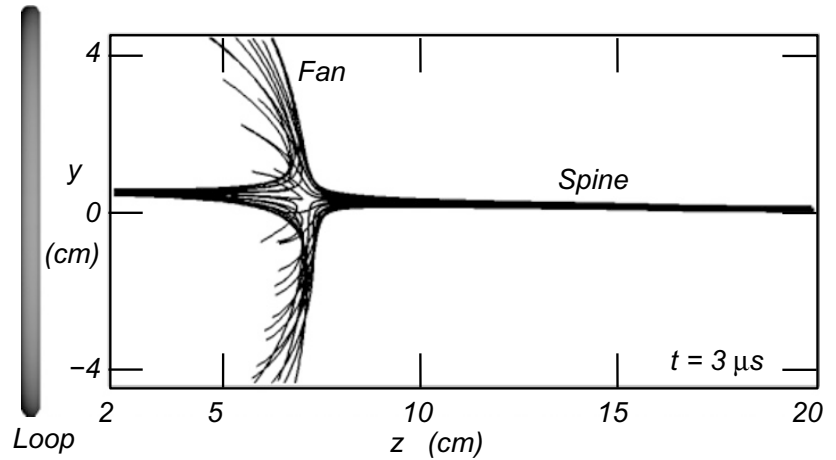

(b)

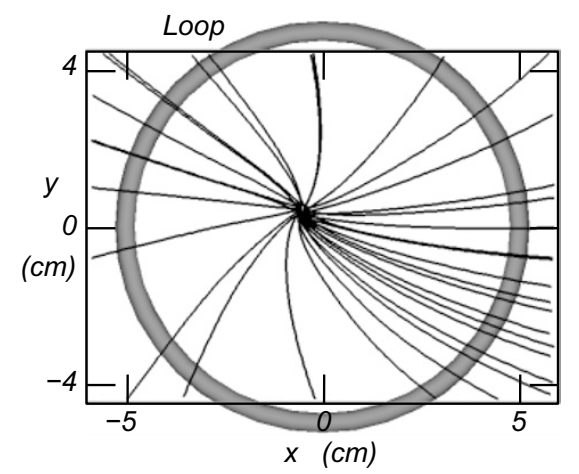

(c)

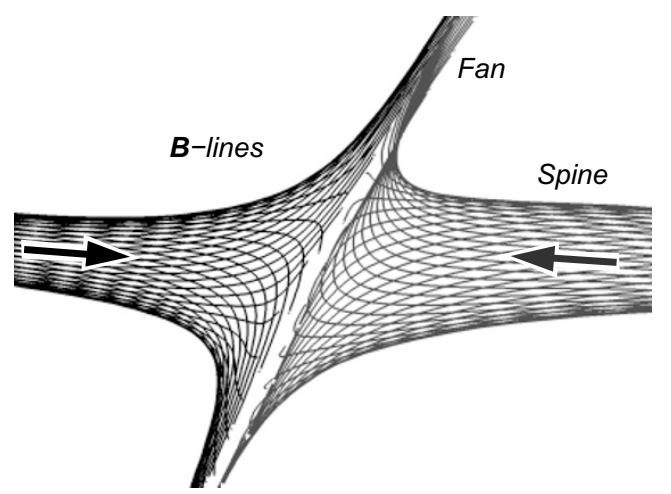

(d)

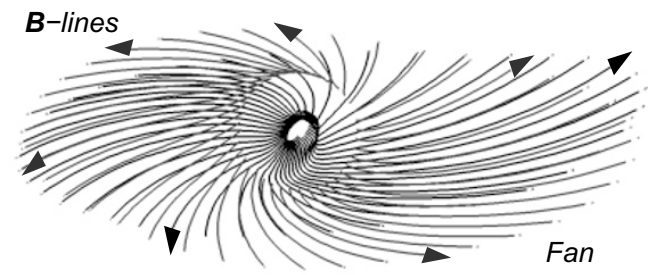

Fig. 5. Measured field lines traced through a 3D null point. $(a, b)$ Cusp-type 3D null point dominated by the potential field of the antenna. (c, d) Improper spiral null established by plasma currents $\left(I_{\text {antenna }}=0\right)$.

ments on driven EMHD reconnection (Stenzel et al., 1986) have neither produced current sheets of thickness $\leq c / \omega_{p e}$. 3.2 Nonlinear EMHD vortices with spiral null points

In these experiments, a large current $(\leq 250 \mathrm{~A})$ is pulsed through a loop antenna (15 cm diam, 4 turns) and maintained sufficiently long ( $\Delta t \simeq 5 \mu \mathrm{s}$ ) to establish a potential field with two cusp-type null points on axis and an O-type null line inside the wire. Figure 3(a) shows schematically the field topology and Fig. 3(b) the typical waveform of the applied current. The loop current is abruptly switched off $\left(\Delta t_{\text {fall }} \simeq 0.75 \mu \mathrm{s}\right)$ and the free relaxation of the initial field topology is measured. Figure 4 shows vector fields of the total magnetic field, $\mathbf{B}_{\text {tot }}=\mathbf{B}_{\text {antenna }}+\mathbf{B}_{\text {plasma }}+\mathbf{B}_{0}$, both during the applied current pulse (a) and after the end of the current pulse $(b, c)$ when $\mathbf{B}_{\text {antenna }}=0$. In contrast to linear vortices, nonlinear vortices do not split up into two oppositely propagating vortices. This is due to the null points inhibiting the axial propagation of whistlers ( $v_{\text {group }} \rightarrow 0$ as $\left.\omega / \omega_{c e} \geq 1\right)$. The vortex is observed to slowly stretch axially and then to shrink as more and more closed field lines are reconnected to open field lines. The toroidal field (not shown in Fig. 4) has opposite sign at each end of the vortex while vanishing at the center, i.e., it is a Siamese twin vortex with zero net helicity. The null points are characterized as improper spiral nulls with twisted fan lines (due to currents along the spine) and a fan plane oblique to the spine axis (due to currents across the spine). Examples of field lines traced through the null points are shown in Fig. 5. During the constant part of the applied current pulse (Figs. 5(a), (b)) one observes essentially the cusp-type null point of a potential field, but after the current switch-off (Figs. 5(c), (d)) the plasma currents deform it into an improper spiral null. The plasma currents are distributed and do not form narrow current sheets. Within $\Delta t \lesssim 5 \mu$ s after switch-off, all the closed poloidal field lines have been reconnected, the O-line and the spiral nulls coalesced, and the topology changed to contain only open (although twisted and bent) field lines. The field line perturbations can now propagate away in the whistler mode.

\subsection{Reconnection rate and particle energization}

The rate of flux transfer across the separatrix provides one definition for the reconnection rate (Vasyliunas, 1975). It can be measured by the flux change through the loop, which is linked with closed poloidal field lines. Figure 6(a) shows the magnetic flux $\Phi(t)=-\int_{0}^{t} V_{\text {loop }}\left(t^{\prime}\right) d t^{\prime}$ in both vacuum and plasma. In vacuum, the flux varies like the applied current, but in the plasma the induced plasma currents slow down the rise and fall of the flux. After switch-off of the antenna current, the open-loop voltage yields the approximate reconnection rate as long as the vortex does not propagate away from the loop. The measured typical reconnection rate is $d \Phi / d t=-V_{\text {loop }} / N_{\text {turns }} \simeq 5 \mathrm{~V}$, which yields an inductive electric field $E_{\theta} \simeq 0.1 \mathrm{~V} / \mathrm{cm}$ along the neutral line. For a typical current density $J_{\theta} \simeq 2 \mathrm{~A} / \mathrm{cm}^{2}$, one obtains a plasma conductivity $\sigma_{\|}=J_{\theta} / E_{\theta} \simeq 20 \Omega^{-1} \mathrm{~cm}^{-1}$, which is consistent with the classical conductivity $\sigma_{\text {Spitzer }}=$ $n e^{2} / m v_{e i} \simeq 28 \Omega^{-1} \mathrm{~cm}^{-1}$ for $k T_{e} \simeq 2 \mathrm{eV}$. Thus, no significant anomalous resistivity is involved in this spontaneous reconnection event. Probe traces also exhibit no high frequency density fluctuations $\left(\omega \leq \omega_{p i}\right)$. These results differ from many other reconnection experiments where strong inductive electric fields are imposed by external time-varying magnetic fields rather than by the self-consistently decaying fields of plasma currents.

During the reconnection process, magnetic energy of the vortex field is dissipated into kinetic energy, predominantly of the electrons, partially of ions. Langmuir probe measurements show a doubling of the electron temperature in the region of the vortex. Tail particles in the heated electron distribution have sufficient energy $(\gtrsim 10 \mathrm{eV})$ to excite transitions in inelastic electron-neutral and ion collisions. The light is detected with a photodiode and displayed in Fig. 6(b) 

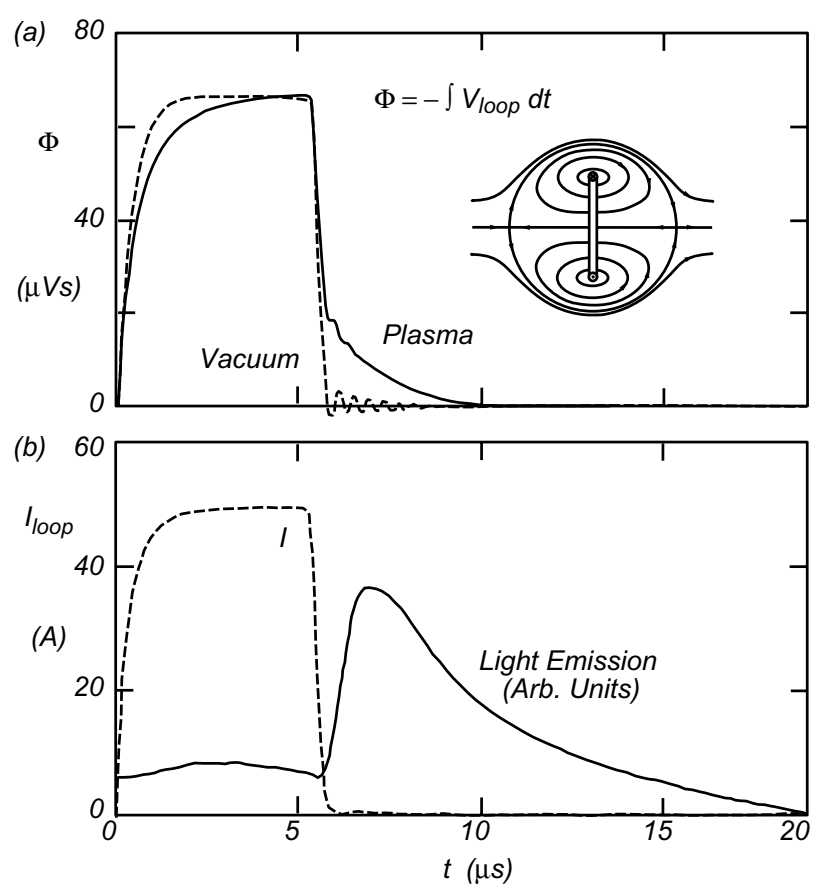

Fig. 6. Reconnection rate and energy conversion. (a) Magnetic flux $\Phi(t)$ of closed field lines (see sketch) in vacuum and plasma. The reconnection of the EMHD vortex occurs at a rate $|\Phi(t) / d t| \lesssim 5 \mathrm{~V}$. (b) Light emission produced by heated electrons and, for timing reference, the applied antenna current. Note that electron energization occurs after the end of the current pulse, i.e., during the reconnection of the $3 \mathrm{D}$ vortex.

in comparison with the waveform of the antenna current. It is evident that light is produced mainly after the turn-off of the applied current pulse, i.e., during the reconnection of the vortex in the presence of null points. During the rise of the antenna current, the induced plasma currents are mainly Hall currents, which convect rather than dissipate magnetic energy. Reversal of the sign of the antenna current pulse produces negligible light since no null line is formed. Thus, electron heating and associated light emission is clearly a result of reconnection. It should also be mentioned that an outward propagating density perturbation $(\delta n /\langle n\rangle \lesssim 20 \%)$ has been observed. Since its initial speed exceeds the sound speed, it is not a wave phenomenon, but a burst of ions accelerated by a radial electric field. However, the ion energization is likely due to the larger applied electric field rather than the smaller self-consistent field during the decaying vortex.

\subsection{Merging of EMHD vortices into an EMHD FRC}

Two nonlinear vortices are generated with identical pulses and antennas (10 cm diam) separated by $\Delta z=25 \mathrm{~cm}$ along $\mathbf{B}_{0}$. Figure 7 shows the vortices (a) schematically and (b) experimentally. During their relaxation the two vortices are observed to merge into a single, elongated, field-reverse configuration (EMHD-FRC) as sketched in Fig. 7(c). This change in field topology implies that magnetic field line reconnection takes place. Figures 8 and 9 display measured vector fields of $\mathbf{B}_{\text {tot }} / B_{\text {tot }}$ in the transverse $y-z$ plane $(x=0)$ at a few characteristic times showing different field topologies. Figure 8(a) shows two distinct vortices prior to the turn-off of the antenna current. After the simultaneous (a)
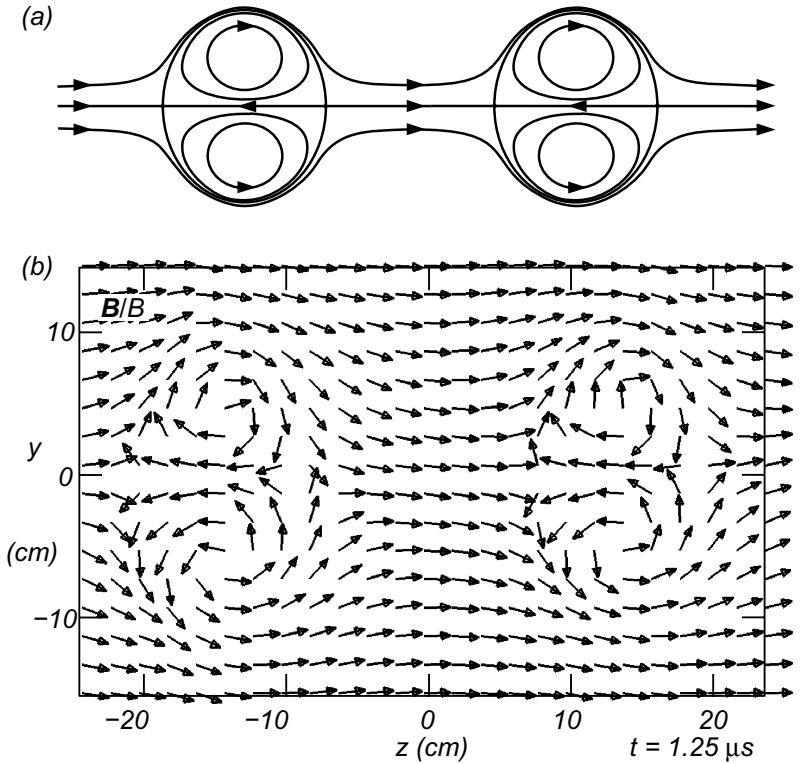

(c)

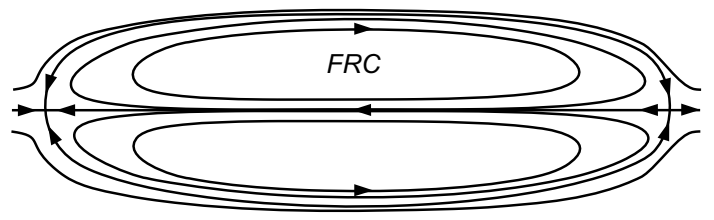

Fig. 7. Merging of two EMHD vortices into a field-reversed configuration (FRC). (a) Schematic of two initial vortices. (b) Unit vector fields of observed vortices. (c) Schematic of final FRC.

switch-off of the loop currents at $t \simeq 5 \mu \mathrm{s}$, the vortices tilt and expand, whereby the two adjacent null points merge first into a single degenerate 3D null point that has two conical separatrix surfaces (Fig. 8(b)). This higher-order null point is topologically unstable (Bulanov et al., 1996; Parnell et al., 1996) and rapidly changes into a 2D null line. In the cut plane shown in Fig. 8(c), the null line exhibits two X-type nulls, while the null lines through the center of the vortices have O-type nulls. All null lines are tilted. [It is interesting to note that these topological changes are the time reversal of the driven reconnection processes that occur during the turnon phase of the antenna current pulse. There, with increasing loop current, the $2 \mathrm{D}$ null line inside the loop collapses to a degenerate null point at the center of the loop, and subsequently splits into two regular 3D null points on axis.] In the present spontaneous merging process, the newly formed null line merges with the null lines of the two vortices to form a long cylindrical current layer whose solenoidal field is stronger than $B_{0}$, and creates a net reverse field at the center (Fig. 9(a)). Axially, it is "terminated" by two regular 3D null points (Fig. 9(b)). This elongated vortex has a twice longer lifetime than each individual vortex. It gradually shrinks as the closed vortex flux is reconnected, and the null line and 3D null points eventually merge (Fig. 9(c)). The initial tilt of the field configuration does not increase further during the relaxation process, even though a magnetic dipole antiparallel to a uniform field is not in a stable magnetostatic equilibrium. Although the solenoidal current sheet is not particularly uniform, it does not further tear or produce whistler turbulence or assume small inertial scales, 

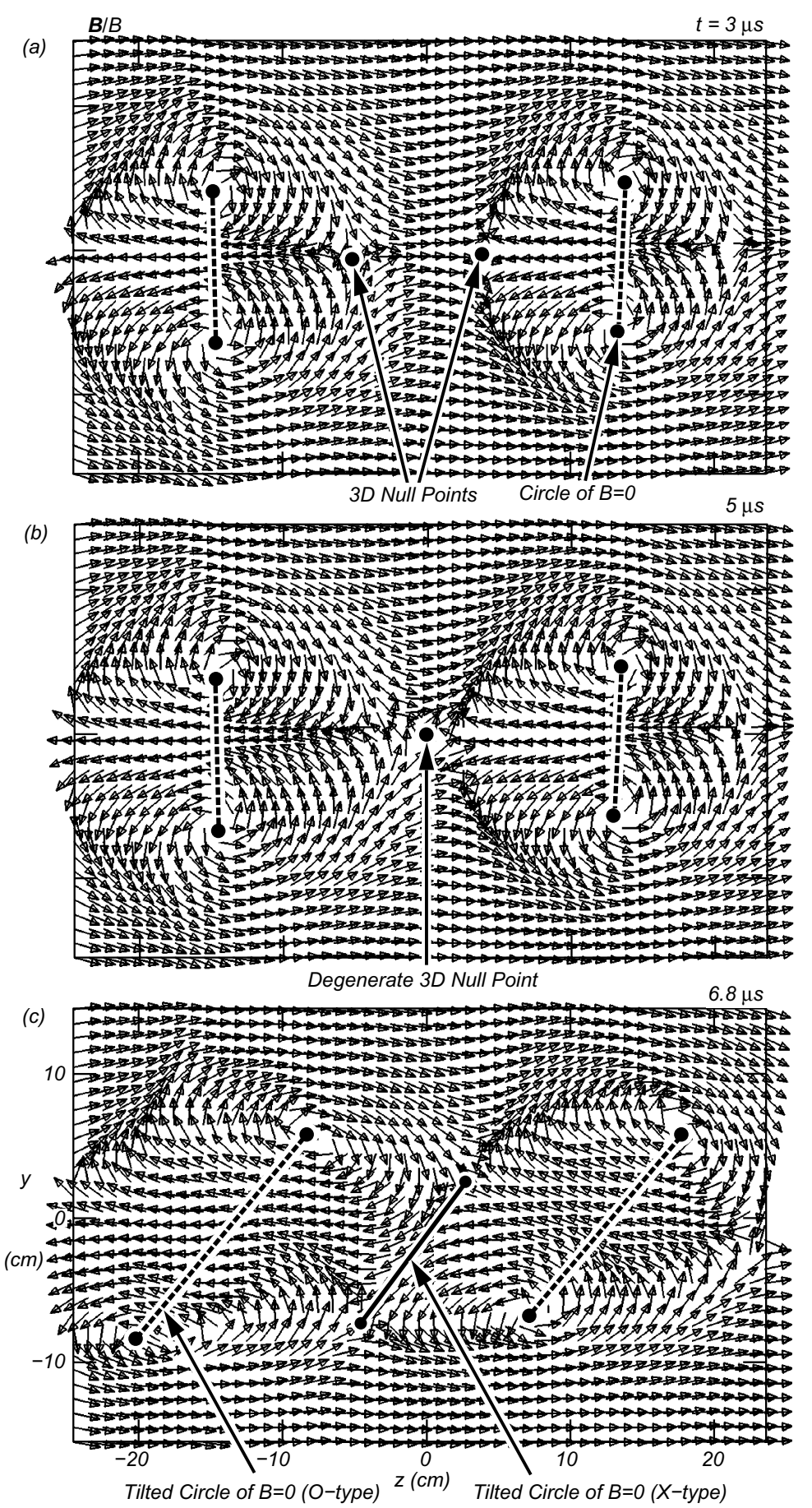

Fig. 8. Measured vector fields $\mathbf{B}_{\text {tot }} / B_{\text {tot }}(0, y, z)$ of two EMHD vortices at different times of their nonlinear interaction. (a) Two separate vortices, each with two 3D null points are created by currents in two loop antennas. (b) At the switch-off of the antenna currents, the vortices tilt, expand, and their adjacent null points merge into a single degenerate null point. (c) The unstable degenerate null point changes into a null line, which in the present cut plane exhibits two X-points.

as has been observed in computer simulations (Biskamp et al., 1996; Drake et al., 1997).

It should be emphasized that the null point topology is mathematically interesting, but that the electrons are essentially unmagnetized at substantial distances $\left(r_{c e}=\right.$ $\left.v_{t h} / \omega_{c e} \geq|B / \nabla B| \approx 5 \mathrm{~cm}\right)$ from the actual null points. Hence, electrons cannot distinguish a null line from a null point. This distance of non-adiabaticity well exceeds the inertial length, $c / \omega_{p e} \simeq 0.75 \mathrm{~cm}$, implying that viscous ef- fects are more important than inertial effects and resistive effects $\left(l_{e i}=v_{t h} / v_{e i} \geq 20 \mathrm{~cm}\right)$.

\section{Summary and Conclusions}

Laboratory experiments have shown how spontaneous reconnection takes place in an unbounded EMHD plasma. The magnetic field topology of vortices has been measured in 3D space and time with resolution comparable to the electron inertial length and cyclotron period. No thin current 


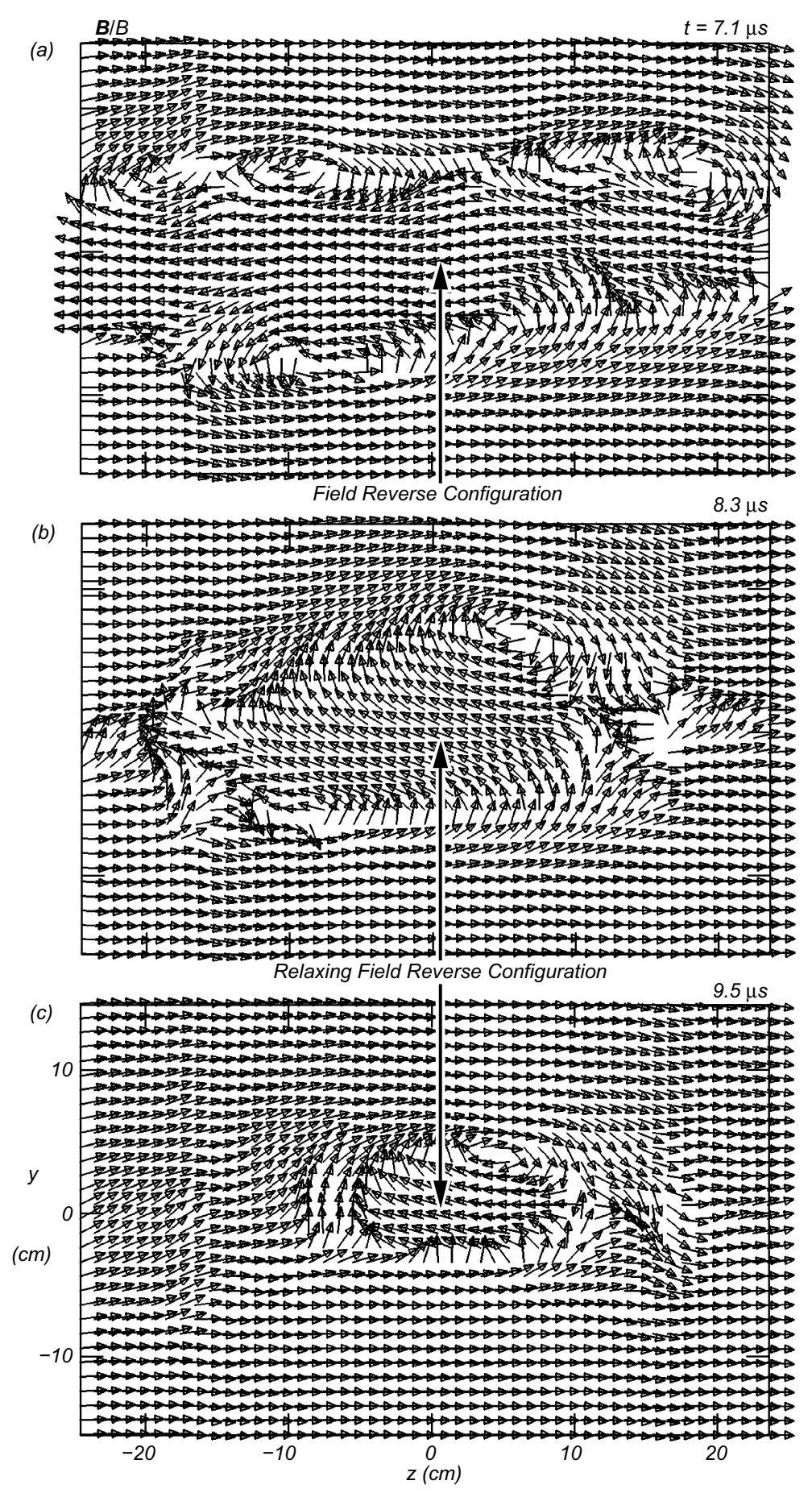

Fig. 9. Measured vector fields $\mathbf{B}_{\text {tot }} / B_{\text {tot }}(0, y, z)$ of two EMHD vortices at different times of their nonlinear interaction. (a) Merging has produced an EMHD-FRC whose central magnetic field is opposite to $\mathbf{B}_{0}$ for $\Delta z>50 \mathrm{~cm}$. (b) and (c) Decaying FRC due to spontaneous reconnection of closed field lines into open ones.

sheets or inertial vortices have been observed. No evidence for whistler turbulence or anomalous resistivity is found. Reconnection near absolute magnetic nulls converts magnetic energy into electron thermal energy. These laboratory observations suggest that in other plasmas of similar parameters (e.g., the solar chromosphere) electron heating by reconnection also occurs, provided EMHD scales are present. The laboratory experiments may further stimulate $3 \mathrm{D} \mathrm{nu}-$ merical simulations in order to resolve the apparent discrepancy between the predicted inertial-scale current sheets from simulations versus the lack of such observations in all present laboratory experiments.

Acknowledgments. The authors gratefully acknowledge support for this work from NSF grant PHY 9713240.

\section{References}

Biskamp, D., E. Schwarz, and J. F. Drake, Two-dimensional electron magnetohydrodynamic turbulence, Phys. Rev. Lett., 76, 1264-1267, 1996.

Bulanov, S. V., V. V. Pichushkin, and K. Schindler, Magnetic field line reconnection in structurally unstable magnetic configurations, Plasma 
Physics Reports, 22, 885-896, 1996.

Drake, J. F., D. Biskamp, and A. Zeiler, Breakup of the electron current layer during 3-D collisionless magnetic reconnection, Geophys. Res. Lett., 24, 2921-2924, 1997.

Ji, H., M. Yamada, R. Kulsrud, N. Pomphrey, and H. Himura, Studies of global stability of field-reversed configuration plasmas using rigid body model, Phys. Plasmas, 5, 3685-3693, 1998.

Kingsep, A. S., K. V. Chukbar, and V. V. Yan'kov, Electron magnetohydrodynamics, in Reviews of Plasma Physics, 16, edited by B. B. Kadomtsev, pp. 243-291, Consultants Bureau, New York, 1990.

Parnell, C. E., J. M. Smith, T. Neukirch, and E. R. Priest, The structure of three-dimensional magnetic null points, Phys. Plasmas, 3, 759-770, 1996.

Rousculp, C. L., R. L. Stenzel, and J. M. Urrutia, Pulsed currents carried by whistlers. V: Detailed new results of magnetic antenna excitation, Phys. Plasmas, 2, 4083-4093, 1995.

Stenzel, R. L. and J. M. Urrutia, Force-free electromagnetic pulses in a laboratory plasma, Phys. Rev. Lett., 65, 2011-2014, 1990.

Stenzel, R. L. and J. M. Urrutia, Laboratory studies of magnetic vortices. I. Directional radiation of whistler waves based on helicity injection, Phys. Plasmas, 6, 2989-2996, 1999.
Stenzel, R. L., W. Gekelman, and N. Wild, Magnetic field line reconnection experiments. IV. Resistivity, heating, and energy flow, J. Geophys. Res., 85, 111-117, 1982.

Stenzel, R. L., W. Gekelman, and J. M. Urrutia, Lessons from laboratory experiments on reconnection, Adv. Space Res., 6, 135-147, 1986.

Stenzel, R. L., J. M. Urrutia, and M. C. Griskey, Laboratory studies of magnetic vortices. II. Helicity reversal during reflection of a magnetic vortex at a conducting boundary, Phys. Plasmas, 6, 4458-4466, 1999.

Urrutia, J. M., R. L. Stenzel, and C. L. Rousculp, Pulsed currents carried by whistlers. II. Excitation by biased electrodes, Phys. Plasmas, 1, 1432$1438,1994$.

Urrutia, J. M., R. L. Stenzel, and M. C. Griskey, Laboratory studies of magnetic vortices. III. Collisions of electron magnetohydrodynamic vortices, Phys. Plasmas, 7, 519-528, 2000.

Vasyliunas, V. M., Theoretical models of magnetic field line merging, I, Rev. Geophys. Space Phys., 13, 303-336, 1975.

R. L. Stenzel (e-mail: stenzel@physics.ucla.edu), J. M. Urrutia, M. C. Griskey, and K. D. Strohmaier 